\title{
Different Signaling Pathways Mediate Regenerative versus Developmental Sensory Axon Growth
}

\author{
Rong-Yu Liu and William D. Snider \\ Neuroscience Center, University of North Carolina, Chapel Hill, North Carolina 27599
}

Recent advances in defining neurotrophin signaling mediators have provided insights into the signal transduction mechanisms that underlie axon growth. Evidence is accumulating that major Trk effectors regulate the morphological development of embryonic peripheral neurons. Less is known about signaling related to the robust axon extension that follows peripheral axotomy of adult neurons. Regenerative axon growth can be mimicked in vitro by a "conditioning" lesion performed 2 weeks before culture (Smith and Skene, 1997). Previous work has implicated both neurotrophins and cytokines in this response. Because signal transduction mediators of both of these families of growth factors are well characterized, we have compared the role of neurotrophin and cytokine signaling in developmental versus regenerative sensory axon growth.

Chemical inhibitors were administrated to embryonic and axotomized sensory neurons in vitro to block the activation of Erk kinase (MEK)-extracellular signal-regulated kinase (ERK), phosphatidylinositol-3 kinase (PI3-K), and janus kinase (JAK)

Recent advances in the study of neuronal signal transduction have yielded new insights into the regulation of neuronal morphology. The most carefully studied paradigms involve mediators of neurotrophin signaling. In the peripheral nervous system (PNS), NGF and related members of the neurotrophin family regulate axon growth during embryonic development (Patel et al., 2000; Tucker et al., 2001) (for review, see Bibel and Barde, 2000). Most current evidence is consistent with the idea that Trk phosphorylation triggers extracellular signal-related kinase (ERK) activation that is then instructive for morphological responses (Atwal et al., 2000; for review, see Kaplan and Miller, 2000). A second major pathway activated by Trk phosphorylation, phosphatidylinositol-3 kinase (PI3-K), is also required for axon growth, but this effect may be related to a requirement for PI3-K in retrograde signaling (Atwal et al., 2000; Kuruvilla et al., 2000). Many non-neurotrophin growth factors such as cytokines, glial cell-derived neurotrophic factor (GDNF) family members, and hepatocyte growth factor are also expressed in the pathways of developing peripheral neurons (for review, see Murphy et al., 1997; Maina and Klein, 1999; Baloh et al., 2000). However,

\footnotetext{
Received May 11, 2001; revised June 19, 2001; accepted June 28, 2001.

This work was supported by National Institute of Neurological Disorders and Stroke Grant NS31768. We thank Lyanna Li for assistance with the initial experiments. Annette Markus, Dan Fisher, Fengquan Zhou, and Patricia Maness made helpful comments on this manuscript.

Correspondence should be addressed to William D. Snider, Neuroscience Center, Campus Box 7250, University of North Carolina, Chapel Hill, NC 27599. E-mail: wsnider@med.unc.edu.

Copyright (C) 2001 Society for Neuroscience $\quad 0270-6474 / 01 / 210001-05 \$ 15.00 / 0$
}

signaling. As expected, both MEK and PI3-K inhibition blocked axon growth from both naïve and NGF-stimulated embryonic day 13 sensory neurons, whereas inhibition of JAK phosphorylation had no effect. In contrast, neither MEK nor PI3-K inhibitors blocked elongation of adult sensory neurons after a conditioning lesion. However, the addition of a JAK2 inhibitor prevented the regenerative axon response. Consistent with these pharmacological results, the percentage of neurons showing intense nuclear signal transducers and activators of transcription 3 phosphorylation after a conditioning lesion was markedly increased compared with controls.

These observations demonstrate that the signaling mediators that underlie regenerative axon growth are distinct from those used during development and suggest that cytokine signaling may be critical to peripheral nervous system regeneration.

Key words: axotomy; neurotrophin signaling; cytokine signaling; conditioning lesion; embryonic axon growth

morphological regulation by these non-neurotrophin growth factors during development is poorly characterized.

Axotomy of adult PNS neurons induces rapid axon regeneration. Far less is known about growth factor signaling mediators of this morphological response of mature neurons. A popular idea is that axotomy induces neurons to "dedifferentiate" and access embryonic growth programs (Aubert et al., 1995; Smith and Skene, 1997). Consistent with this idea, expression of various neuronal growth factors, including NGF and GDNF family members and neuroactive cytokines, is upregulated by Schwann cells in distal peripheral nerve after transection (Heumann et al., 1987; Curtis et al., 1994; Naveilhan et al., 1997). It is believed, but not yet proven, that these growth factors play an important role in successful PNS regeneration [however, see Diamond et al. (1992); for review, see Zigmond et al., 1996; Terenghi, 1999]. It therefore seems logical to ask whether the growth factor signaling mediators that underlie the regenerative axon growth of adult neurons

This article is published in The Journal of Neuroscience, Rapid Communications Section, which publishes brief, peerreviewed papers online, not in print. Rapid Communications are posted online approximately one month earlier than they would appear if printed. They are listed in the Table of Contents of the next open issue of JNeurosci. Cite this article as: JNeurosci, 2001, 21:RC164 (1-5). The publication date is the date of posting online at www.jneurosci.org.

http://www.jneurosci.org/cgi/content/full/5611 
are the same or different from the mediators of embryonic axon growth.

The axon regeneration response of mature neurons can be modeled in vitro for studies of signaling mechanisms by a "conditioning" lesion of nerve transection 1-2 weeks before culture (Smith and Skene, 1997; Lankford et al., 1998, Neumann and Woolf, 1999; Bomze et al., 2001). Under these circumstances, axon elongation occurs rapidly over $24-48 \mathrm{hr}$ in vitro. To study the signal transduction pathways that mediate axon elongation, pharmacological inhibitors were added to embryonic and adult mouse DRG cultures for blockade of MEK-ERK, PI3-K, protein kinase A (PKA), and janus kinase (JAK)/signal transducers and activators of transcription (STAT) signal transduction pathways. Here we show that although MEK and PI3-K inhibitors strongly reduce axon outgrowth from embryonic neurons in response to NGF, they have no effect on axon extension after a conditioning lesion in maturity. PKA inhibition is without effect on either embryonic or adult neurons. However, an inhibitor of cytokine signaling that has no effect on embryonic axon growth, the JAK2 inhibitor AG490, markedly inhibits axon extension after a conditioning lesion. Consistent with this latter finding, immunohistochemistry showed phosphorylation and nuclear translocation of the cytokine activated transcription factor STAT3 in preaxotomized neurons. Our results demonstrate that JAK/STAT rather than ERK signaling mediates the rapid extension of adult sensory axons induced by previous axotomy.

\section{MATERIALS AND METHODS}

Embryonic DRG neuron culture. Highly dissociated DRG cultures from embryonic day 13 (E13) Bax ${ }^{-1-}$ mice were prepared as described previously (Lentz et al., 1999). Dissociated neurons were plated on glass coverslips (Thomas Scientific) coated with a mixture of poly-D-lysine (0.1 $\mathrm{mg} / \mathrm{ml}$; Sigma, St. Louis, MO) and laminin (4 ng/ml; Collaborative Biomedical Products, Bedford, MA) at 2000 cells per coverslip. Cultures were maintained in Minimal Essential Medium (MEM; Life Technologies, Gaithersburg, MD) containing 5\% HI-FBS, 2 mM L-glutamine, and $10 \mu \mathrm{M}$ uridine/5-fluoro-2'-deoxyuridine (Sigma). Some cultures were supplemented with NGF at $50 \mathrm{ng} / \mathrm{ml}$. Well characterized pharmacological inhibitors of MEK (U0126; Promega, Madison, WI), PI3-K (LY294002; Calbiochem, La Jolla, CA), PKA (KT5720, Calbiochem), and JAK2 (AG490, Calbiochem) were added to the cultures at the time of plating. Each experiment was repeated with embryos from four separate matings.

Sciatic nerve transection and adult DRG culture. Adult CF1 mice were anesthetized by intraperitoneal injection of $2.5 \%$ Avertin (Sigma). Both sciatic nerves were transected. Mice were allowed to survive for 7-14 d. Then the fourth and fifth lumbar (L4 and L5) DRGs were removed. Cells were dissociated enzymatically with collagenase A for $1.5 \mathrm{~h}$ followed by trypsin/EDTA for $15 \mathrm{~min}$. Adult DRG neuronal culture was obtained by centrifugation and resuspension twice through MEM containing 10\% FBS to remove myelin debris (Smith and Skene, 1997). Cells were resuspended in MEM with addition of 5\% HI-FBS, 2 mM L-glutamine, and $25 \mu \mathrm{M}$ uridine/5-fuloro-2'-deoxyuridine. Dissociated L4/15 neurons were plated onto coverslips coated as above at the concentration of 1000 cells per coverslip. This appeared to be the optimal plating density because the axonal arbors of many neurons could be traced in their entirety. At lower densities, high concentrations of U0126 appeared to cause neuronal damage as manifested by axon blebbing. NGF $(50 \mathrm{ng} / \mathrm{ml})$ and/or the pharmacological inhibitors described above were added to the cultures 1-2 hr later when medium was changed to eliminate cell debris. Adult DRG neurons from C57BL/6 mice exhibited qualitatively similar responses to the conditioning lesion and to NGF.

Immunochemistry. To visualize DRG axons, cultures were stained with a monoclonal antibody (SMI 31) directed against phosphorylated neurofilament $\mathrm{H}$ and $\mathrm{M}$ subunits as described previously. To assess STAT3 activation, neurons were cultured as described above for $18 \mathrm{hr}$, then incubated in serum-free medium with or without $50 \mu \mathrm{M}$ AG490 for $6 \mathrm{hr}$. Cells were incubated with a polyclonal antibody against phospho-STAT3 (Tyr705; Cell Signaling). The immunocytochemistry protocols were

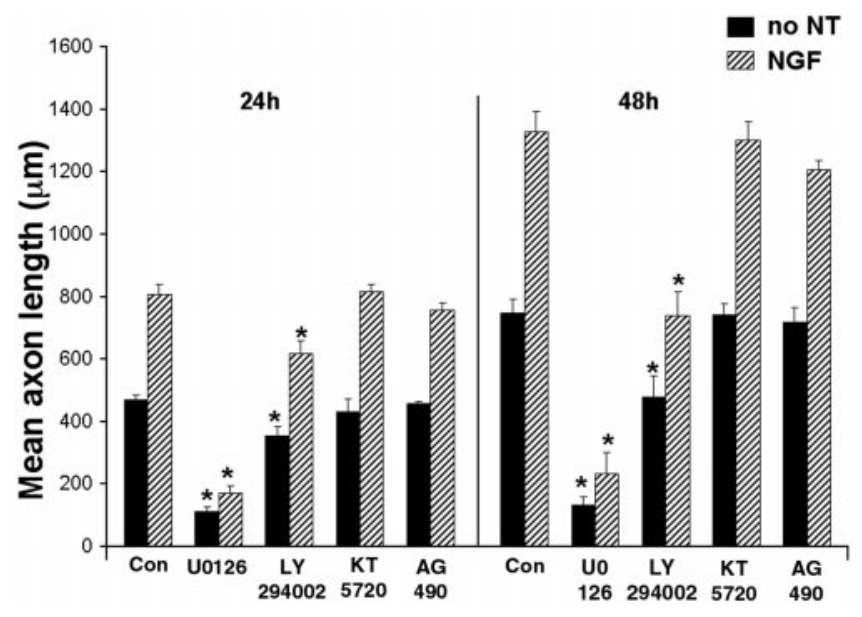

Figure 1. Effects of signal transduction inhibitors on axon growth from E13 $\mathrm{Bax}^{-/-}$DRG neurons. Bars show mean \pm SEM from four experiments with each inhibitor. ${ }^{*} p<0.05$.

provided by the manufacturer. To quantify pSTAT3 nuclear labeling density, images of at least 50 neurons in each experiment were digitally captured using a Spot II camera, then analyzed using densitometry software (IP Lab).

Quantification of axon length. Neurons were traced with camera lucida at $100 \times$ using bright-field optics. For each condition in each experiment, all neurons that were distinguishable from neighboring neurons were drawn until 30-50 neurons were collected. The drawings were then scanned into a computer, and axon lengths were quantified using NIH Image (1.61). For E13 and adult neurons cultured for $24 \mathrm{hr}$, total axon lengths were measured. By $48 \mathrm{hr}$, axonal arbors from adult NGF-treated and condition-lesioned animals were too complex for full tracing. Therefore, at this time point, the length of the longest elaborated axon was determined.

\section{RESULTS}

\section{Signaling pathways regulating developmental axon growth}

Most current evidence is consistent with the idea that both ERK and PI3-kinase pathways are required for the embryonic axon growth observed in the presence of neurotrophins (Klesse and Parada, 1998; Atwal et al., 2000; Kaplan and Miller, 2000). However, previous studies have been complicated by the absolute requirement for neurotrophins for peripheral neuron survival during development. To separate morphological from survival effects of signal transduction mediators, E13 DRG neurons from mutant mice lacking the proapoptotic protein BAX were cultured. Normally, E13 Bax-null neurons respond to neurotrophins with rapid axon elongation over $48 \mathrm{hr}$ on a favorable substratum (Lentz et al., 1999). We tested axon growth in the presence of the membrane-permeable inhibitors U0126, LY294002, KT5720, and AG490, which are specific for MEK, PI3-K, PKA, and JAK2, respectively.

As expected, the MEK inhibitor, U0126, almost completely blocked baseline and NGF-induced axon extension. U0126 (10 $\mu \mathrm{M}$ ) decreased total axon length fivefold at 24 and $48 \mathrm{hr}$ (Fig. 1) without killing the $\mathrm{Bax}^{-/-}$neurons. LY294002, a PI3-K inhibitor, also inhibited baseline and NGF-induced axon extension, again without killing the neurons. The effect of LY294002 was more pronounced at 48 than $24 \mathrm{hr}$. Addition of $800 \mathrm{~nm}$ KT5720 to inhibit PKA did not change axon length significantly either at baseline or in response to NGF. Similarly, addition of $50 \mu \mathrm{M}$ AG490 to inhibit JAK2 had no effect (Fig. 1). 


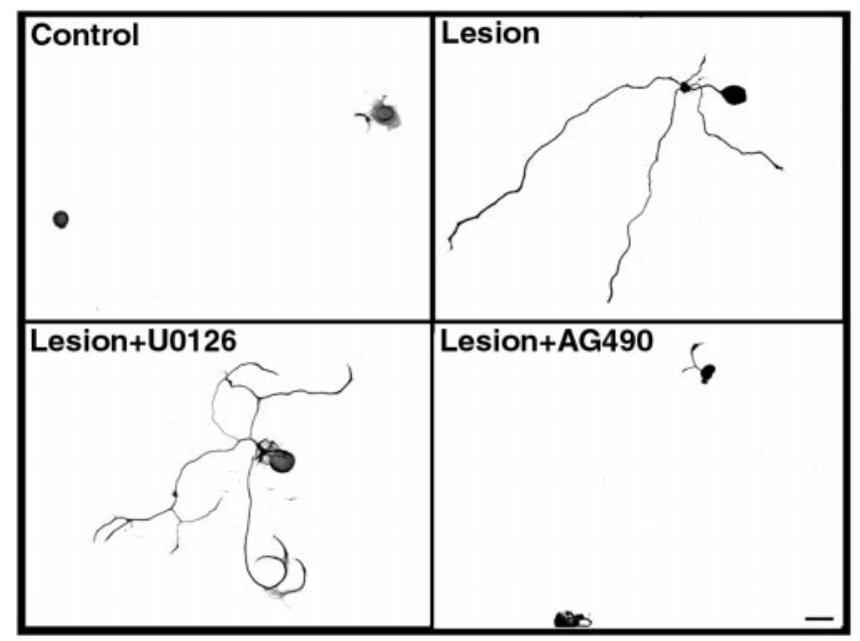

Figure 2. Representative axonal morphologies from control and conditioned adult DRG neurons at $24 \mathrm{hr}$ in culture. Axon growth is markedly inhibited by AG490, whereas UO126 has no noticeable effects. Scale bar, $50 \mu \mathrm{m}$.
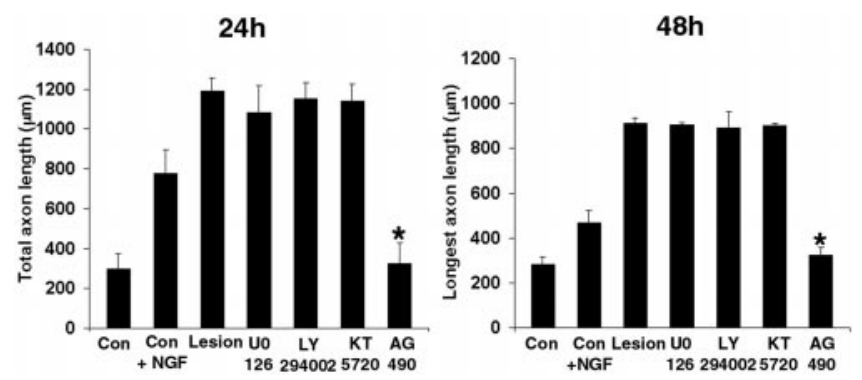

Figure 3. Effects of signal transduction inhibitors on axon growth from control and conditioning-lesioned adult DRG neurons. Total axon length was determined at $24 \mathrm{hr}$, and length of the longest process was determined at $48 \mathrm{hr}$. Bars show mean \pm SEM from four experiments. ${ }^{*} p<0.01$.

\section{Signaling pathways regulating regenerative axon outgrowth}

As reported previously (Smith and Skene, 1997), few adult DRG neurons from control mice extend axons within the first $24 \mathrm{hr}$ in culture (Fig. 2), and growth is highly branched after $24 \mathrm{hr}$ (data not shown). In contrast, previous axotomy (conditioning lesion) results in rapid axon extension beginning within $2-3 \mathrm{hr}$ and reaches a mean of $\sim 1200 \mu \mathrm{m}$ in total length by $24 \mathrm{hr}$ (Figs. 2, 3). Significant axon growth at $24 \mathrm{hr}$ is exhibited by $\sim 60 \%$ of neurons after a conditioning lesion compared with $<10 \%$ of controls. Note that this axon extension occurs in the absence of exogenous growth factors. Addition of NGF enhanced axon growth of some neurons in terms of total axon length (Fig. 3) but did not mimic the effects of the conditioning lesion because NGF-treated neurons remained highly branched (data not shown).

Addition of pharmacological inhibitors of signal transduction had very different effects on axon growth in mature as compared with embryonic neurons. Addition of either $25 \mu \mathrm{M}$ U0126 or 50 $\mu \mathrm{M}$ LY294002 abolished NGF-induced morphological responses (data not shown). However, neither compound had any effect on axotomy-induced axon extension at 24 or $48 \mathrm{hr}$ (Figs. 2, 3). At a concentration of $800 \mathrm{nM}$, fourfold of the reported effective concentration (Boulanger and Poo, 1999; Cai et al., 1999), the PKA inhibitor KT5720 similarly had no effect on the length of previously axotomized neurons.

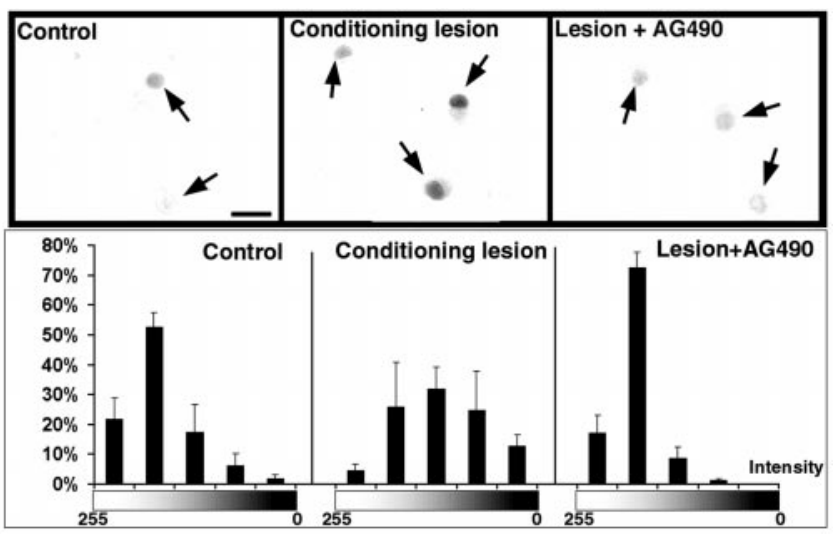

Figure 4. Immunostaining for phospho-STAT3. Activation of STAT3 was increased in the nucleus of DRG neurons after a conditioning lesion. Activation of STAT3 was markedly decreased after addition of AG490. Scale bar, $20 \mu \mathrm{m}$.

In striking contrast, AG490, at a concentration of $50 \mu \mathrm{M}$, strongly inhibited axon elongation induced by a conditioning lesion at 24 and $48 \mathrm{hr}$ (Figs. 2, 3). After a conditioning lesion, DRG neurons exhibited between 200 and $3000 \mu \mathrm{m}$ of total axon length at $24 \mathrm{hr}$, with $\sim 60 \%$ of neurons showing total axon length $>600 \mu \mathrm{m}$. In contrast, in cultures treated with AG490, $\sim 90 \%$ of neurons exhibited total axon lengths of $<600 \mu \mathrm{m}$ at $24 \mathrm{hr}$. The magnitude of inhibition attributable to AG490 was similar at 48 $\mathrm{hr}$; however, we quantified this as length of the longest axon because of the complexity of axon growth from NGF-treated and conditioning-lesioned neurons at this time point. AG490 did not have any noticeable effect on adult neuron survival.

To determine whether the JAK/STAT pathway was activated in conditioning-lesioned neurons, cultures were stained with an antibody against phosphorylated STAT3. DRG neurons from conditioning-lesioned mice showed intense nuclear labeling of phosphorylated STAT3, compared with controls (Fig. 4). Densitometry revealed that $\sim 80 \%$ of neurons in conditioned lesion cultures showed staining above the mean value for control neurons. pSTAT3 labeling was dramatically reduced after AG490 treatment.

\section{DISCUSSION}

Recent studies on neurotrophin signal transduction have indicated that the Trk effector pathways, ERK and PI3-K, are required for growth of embryonic peripheral axons (for review, see Kaplan and Miller, 2000). However, previous studies inhibiting MEK and PI3-K have been restricted to the growth of distal axons into an NGF-containing compartment in Campenot chambers because of the survival dependence of peripheral neurons on neurotrophins (Atwal et al., 2000; Kuruvilla et al., 2000). Our results using $\mathrm{Bax}^{-/-}$neurons that do not require neurotrophin signaling for survival demonstrate the detailed axonal morphology of dissociated sensory neurons under conditions of pharmacological inhibition of neurotrophin signaling mediators. We show here that the ERK pathway is absolutely required for axon extension on laminin in the absence of NGF and in the setting of NGF stimulation. Consistent with the results of Atwal et al. (2000), we find that the PI3-K pathway is also required for normal axon growth, although some NGF-induced axon extension is possible in the presence of LY294002. Finally, activation of the PKA pathway is not required for embryonic sensory axon growth, 
although cAMP stimulates neurite outgrowth from PC12 cells and supports survival of sympathetic and sensory neurons (Rydel and Greene, 1988; Weeks et al., 1991). To our surprise, this pathway also was without effects on adult axon morphology despite the fact that cAMP has been implicated in the ability of neurotrophins to support sensory axon growth over inhibitory substrates and is required for retinal ganglion cells to respond to neuronal growth factors after axotomy (Cai et al., 1999; Shen et al., 1999).

A convenient paradigm for the study of regenerative axon growth of mature peripheral neurons is culture 1-2 weeks after a conditioning axotomy (Smith and Skene, 1997; Lankford et al., 1998; Bomze et al., 2001). Under these circumstances axons extend at a faster rate than controls over the first $48 \mathrm{hr}$ and are significantly less branched. A potential mechanism for regenerative axon growth is that the signal transduction molecules mediating axon growth during development regulate similar morphological changes in maturity. Indeed, the ERK pathway would seem particularly likely to be necessary for regeneration because it is activated by both integrin and cell adhesion molecule signaling (Perron and Bixby, 1999; Schmid et al., 2000). Furthermore, ERK1 mRNA expression is upregulated by axotomy (Mishima et al., 1997). The Akt pathway has also been implicated in regenerative responses because Akt phosphorylation and translocation to the nucleus are induced by axotomy, and expression of membrane-targeted Akt by motor neurons is said to enhance their regenerative response (Namikawa et al., 2000).

Surprisingly, we have found that commonly used inhibitors of MEK and PI3-K have no effect on axon growth induced by a conditioning lesion over the first $48 \mathrm{hr}$ in vitro. The MEK inhibitor was used at concentrations up to 2.5 -fold of those effective in blocking axon growth of embryonic neurons. That the concentrations were effective in inhibiting NGF/Trk signaling was demonstrated by the fact that axon branching in control adult neurons induced by NGF was blocked by these compounds. We cannot conclude that regenerative axon growth in this model is completely ERK independent because ERK-mediated gene transcription may be initiated by lesion-induced neurotrophin expression in peripheral nerve before culturing the neurons. Nevertheless, in contrast to the situation with embryonic neurons, robust axon extension from adult sensory neurons can occur in vitro in the absence of MEK/ERK or PI3-K signaling. Consistent with the concept that different signaling pathways underlie regenerative versus developmental axon growth, another recent study has shown that GAP-43 promoter elements active during development of axon projections in zebrafish are not active during regeneration (Udvadia et al., 2001).

Neuroactive cytokines have been implicated in the regulation of injury-mediated responses in the peripheral nervous system, especially peptide expression (for review, see Zigmond et al., 1996). Expression of LIF and several other neuroactive cytokines is upregulated in distal nerve stump and by satellite cells after a nerve injury (Sun et al., 1996; for review, see Fu and Gordon, 1997). Furthermore, recent work published in abstract form has suggested that LIF enhances NGF-induced axon growth responses of adult DRG neurons in vitro (Cafferty et al., 2000). Finally, knock-out studies have suggested a modest effect of IL6 on regeneration in vivo (Zhong et al., 1999).

Our results demonstrate that axon elongation of adult sensory neurons over the first $24-48 \mathrm{hr}$ in vitro after a conditioning lesion is almost totally blocked by AG490. AG490 is thought to be highly specific for JAK2 (Meydan et al., 1996; Bright et al., 1999;
Yoshikawa et al., 2001). Of course we cannot absolutely exclude the possibility that this compound affects other signaling pathways. An interesting point is that axon elongation after the conditioning lesion does not require cytokines in the medium. Presumably this is related to the fact that STAT3 is activated in the neuronal cultures after a conditioning lesion, possibly caused by cytokine release by satellite cells that cannot be easily removed from the neurons. Also, peripheral axotomy is known to induce increases in expression levels and phosphorylation of several cytokine mediators, including STAT3 (Schwaiger et al., 2000).

Our results demonstrate a link between cytokine signaling and axon growth in adult neurons. The nature of the link between JAK/STAT activation and the neuronal cytoskeleton is unclear at this time. However, there is a precedent for our results in that regulation of B cell morphological responses by STAT6 is well established (Davey et al., 2000). Interestingly a study in PC12 cells that would be expected to model the developmental situation showed that NGF-induced neurite growth was inhibited by activated STAT3 (Ihara et al., 1997). This result again suggests a link between cytokine signaling and axon growth. It is plausible that JAK/STAT signaling is required for the upregulation of gene expression for cytoskeletal elements normally observed after axotomy. This idea should be readily amenable to testing. To our knowledge, local interactions of these cytokine signaling mediators with cytoskeletal elements in neurons have not yet been described.

It is interesting that several neurotrophins and GDNF family members are also upregulated by Schwann cells after a PNS lesion. The role of these factors is unclear. One careful study showed no effect of blocking NGF on the rate of cutaneous nerve regeneration (Diamond et al., 1992). Also, NGF added to the medium tends to cause axon branching rather than elongation of axons [see above and Cafferty et al. (2000)]. It is plausible that neuroactive cytokines and neurotrophins and other factors acting through receptor tyrosine kinases have cooperative actions, regulating different aspects of axon morphology. Robust axon extension and vigorous branching in target fields are both required for successful reinnervation after peripheral nerve injury.

\section{REFERENCES}

Atwal JK, Massie B, Miller FD, Kaplan DR (2000) The Trk-Shc site signals neuronal survival and local axon growth via MEK and PI-3kinase. Neuron 27:265-277.

Aubert I, Ridet JL, Gage FH (1995) Regeneration in the adult mammalian CNS: guided by development. Curr Opin Neurobiol 5:625-635.

Baloh RH, Enomoto H, Johnson Jr EM, Milbrandt J (2000) The GDNF family ligands and receptors: implications for neural development. Curr Opin Neurobiol 10:103-110.

Bibel M, Barde YA (2000) Neurotrophins: key regulators of cell fate and cell shape in the vertebrate nervous system. Genes Dev 14:2919-2937.

Bomze HM, Bulsara KR, Iskandar BJ, Caroni P, Pate Skene JH (2001) Spinal axon regeneration evoked by replacing two growth cone proteins in adult neurons. Nat Neurosci 4:38-43.

Boulanger L, Poo M (1999) Gating of BDNF-induced synaptic potentiation by cAMP. Science 284:1982-1984.

Bright JJ, Du C, Sriram S (1999) Tyrphostin B42 inhibits IL-12-induced tyrosine phosphorylation and activation of Janus kinase-2 and prevents experimental allergic encephalomyelitis. J Immunol 162:6255-6262.

Cafferty WBJ, Gardiner NJ, Thompson SWN (2000) LIF: a conditioning factor that enhances sensory neurone neurite elongation in vitro. Soc Neurosci Abstr 26:65.

Cai D, Shen Y, De Bellard M, Tang S, Filbin MT (1999) Prior exposure to neurotrophins blocks inhibition of axonal regeneration by MAG and myelin via a cAMP-dependent mechanism. Neuron 22:89-101.

Curtis R, Scherer SS, Somogyi R, Adryan KM, Ip NY, Zhu Y, Lindsay RM, DiStefano PS (1994) Retrograde axonal transport of LIF is increased by peripheral nerve injury: correlation with increased LIF expression in distal nerve. Neuron 12:191-204.

Davey EJ, Greicius G, Thyberg J, Severinson E (2000) STAT6 is re- 
quired for the regulation of IL-4-induced cytoskeletal events in B cells. Int Immunol 12:995-1003.

Diamond J, Foerster A, Holmes M, Coughlin M (1992) Sensory nerves in adult rats regenerate and restore sensory function to the skin independently of endogenous NGF. J Neurosci 12:1467-1476.

Fu SY, Gordon T (1997) The cellular and molecular basis of peripheral nerve regeneration. Mol Neurobiol 14:67-116.

Heumann R, Lindholm D, Bandtlow C, Meyer M, Radeke MJ, Misko TP, Shooter E, Thoenen H (1987) Differential regulation of mRNA encoding nerve growth factor and its receptor in rat sciatic nerve during development, degeneration, and regeneration: role of macrophages. Proc Natl Acad Sci USA 84:8735-8739.

Ihara S, Nakajima K, Fukada T, Hibi M, Nagata S, Hirano T, Fukui Y (1997) Dual control of neurite outgrowth by STAT3 and MAP kinase in PC12 cells stimulated with interleukin-6. EMBO J 16:5345-5352.

Kaplan DR, Miller FD (2000) Neurotrophin signal transduction in the nervous system. Curr Opin Neurobiol 10:381-391.

Klesse LJ, Parada LF (1998) p21 ras and phosphatidylinositol-3 kinase are required for survival of wild-type and NF1 mutant sensory neurons. J Neurosci 18:10420-10428.

Kuruvilla R, Ye H, Ginty DD (2000) Spatially and functionally distinct roles of the PI-3-K effector pathway during NGF signaling in sympathetic neurons. Neuron 27:499-512.

Lankford KL, Waxman SG, Kocsis JD (1998) Mechanisms of enhancement of neurite regeneration in vitro following a conditioning sciatic nerve lesion. J Comp Neurol 391:11-29.

Lentz SI, Knudson CM, Korsmeyer SJ, Snider WD (1999) Neurotrophins support the development of diverse sensory axon morphologies. J Neurosci 19:1038-1048.

Maina F, Klein R (1999) Hepatocyte growth factor, a versatile signal for developing neurons. Nat Neurosci 2:213-217.

Meydan N, Grunberger T, Dadi H, Shahar M, Arpaia E, Lapidot Z, Leeder JS, Freedman M, Cohen A, Gazit A, Levitzki A, Roifman CM (1996) Inhibition of acute lymphoblastic leukaemia by a Jak-2 inhibitor. Nature 379:645-648.

Mishima K, Matsuoka H, Yoshikawa T, Ohgushi H, Tabata S, Yamada E (1997) Increased expression of extracellular signal regulated kinase 1 after axotomy in the dorsal motor nucleus of the vagus nerve and the hypoglossal nucleus. Brain Res 766:50-55.

Murphy M, Dutton R, Koblar S, Cheema S, Bartlett P (1997) Cytokines which signal through the LIF receptor and their actions in the nervous system. Prog Neurobiol 52:355-378.

Namikawa K, Honma M, Abe K, Takeda M, Mansure K, Obata T, Miwa A, Okado H, Kiyama H (2000) Akt/protein kinase B prevents injuryinduced motoneuron death and accelerates axonal regeneration. J Neurosci 20:2875-2886.

Naveilhan P, ElShamy WM, Ernfors P (1997). Differential regulation of mRNAs for GDNF and its receptors Ret and GDNFR alpha after sciatic nerve lesion in the mouse. Eur J Neurosci 9:1450-1460.

Neumann S, Woolf CJ (1999) Regeneration of dorsal column fibers into and beyond the lesion site following adult spinal cord injury. Neuron 23:83-91.

Patel TD, Jackman A, Rice FL, Kucera J, Snider WD (2000) Development of sensory neurons in the absence of NGF/TrkA signaling in vivo. Neuron 25:345-357.

Perron JC, Bixby JL (1999) Distinct neurite outgrowth signaling pathways converge on ERK activation. Mol Cell Neurosci 13:3362-3378.

Rydel RE, Greene LA (1988) cAMP analogs promote survival and neurite outgrowth in cultures of rat sympathetic and sensory neurons independently of nerve growth factor. Proc Natl Acad Sci USA 85:1257-1261.

Schmid RS, Pruitt WM, Maness PF (2000) A MAP kinase-signaling pathway mediates neurite outgrowth on L1 and requires Src-dependent endocytosis. J Neurosci 20:4177-4188.

Schwaiger FW, Schmitt GH, Horvat A, Hager G, Streif R, Spitzer C, Gamal S, Breuer S, Brook GA, Nacimiento W, Kreutzberg GW (2000) Peripheral but not central axotomy induces changes in Janus kinases (JAK) and signal transducers and activators of transcription (STAT). Eur J Neurosci 12:1165-1176.

Shen S, Wiemelt AP, McMorris FA, Barres BA (1999) Retinal ganglion cells lose trophic responsiveness after axotomy. Neuron 23:285-295.

Smith DS, Skene JHP (1997) A transcription-dependent switch controls competence of adult neurons for distinct modes of axon growth. J Neurosci 17:646-658.

Sun Y, Landis SC, Zigmond RE (1996) Signals triggering the induction of leukemia inhibitory factor in sympathetic superior cervical ganglia and their nerve trunks after axonal injury. Mol Cell Neurosci 7:152-163.

Terenghi G (1999) Peripheral nerve regeneration and neurotrophic factors. J Anat 194:1-14.

Tucker KL, Meyer M, Barde YA (2001) Neurotrophins are required for nerve growth during development. Nat Neurosci 4:29-37.

Udvadia AJ, Koster RW, Skene JHP (2001) GAP-43 promoter elements in transgenic zebrafish reveal a difference in signals for axon growth during CNS development and regeneration. Development 128:1175-1182

Weeks BS, Papadopoulos V, Kleinman HK (1991) cAMP promotes branching of laminin-induced neuronal processes. J Cell Physiol 147:62-67.

Yoshikawa H, Matsubara K, Oian GS, Jackson P, Groopman JD, Manning JE, Harris CC (2001) SOCS-1, a negative regulator of the JAK/ STAT pathway, is silenced by methylation in human hepatocellular carcinoma and shows growth-suppression activity. Nat Genet 28:29-35.

Zhong J, Dietzel ID, Wahle P, Kopf M, Heumann R (1999) Sensory impairments and delayed regeneration of sensory axons in interleukin6-deficient mice. J Neurosci 19:4305-4313.

Zigmond RE, Hyatt-Sachs H, Mohney RP, Schreiber RC, Shadiack AM, Sun Y, Vaccariello SA (1996) Changes in neuropeptide phenotype after axotomy of adult peripheral neurons and the role of leukemia inhibitory factor. Perspect Dev Neurobiol 4:75-90. 